\title{
REFERENCES
}

1. R. Baer and C. Williams, Splitting criteria and extension types, Bull. Amer. Math. Soc. vol. 55 (1949) pp. 729-743.

2. L. A. Hostinsky, Endomorphisms of lattices, Duke Math. J. vol. 18 (1951) pp. $331-342$.

3. —_ Direct decompositions in lattices, Amer. J. Math. vol. 73 (1951) pp. 741755.

4. - Loewy chains and uniform splitting of lattices, Proc. Amer. Math. Soc. vol. 5 (1954) pp. 315-319.

The Pennsylvania State University

\section{FINITE DIMENSIONAL CENTRAL DIVISION ALGEBRAS}

\section{S. A. AMITSUR}

1. Introduction. Let $D$ be a division algebra over its center $C$. Let $D[x]$ be the ring of all polynomials in a commutative indeterminate $x$ with coefficients in $D$. The present note contains a characterization of the division algebras $D$ which are finite over their center with the aid of the polynomial ring $D[x]$.

One characterization is the following:

Theorem 1. $(D: C) \leqq n^{2}<\infty$ if and only if every primitive homomorphic image of $D[x]$ is a complete matrix ring $A_{h}, h \leqq n$ over a division ring $A$.

With this result at our hand we utilize recent results on pivotal monomials to show:

THEOREM 2. ( $D: C) \leqq n^{2}<\infty$ if and only if for any two polynomials $f(x), g(x) \in D[x]$ the polynomials $1-g(x) f^{n}(x)$ and $f(x)$ do not have a nontrivial left common divisor.

The fact that Theorem 2 gives a characterization of the finite dimensional algebras, means that:

Corollary 1. If $(D: C)>n^{2}$ then $D[x]$ contains two polynomials $g(x), f(x)$ such that $1-g(x) f^{n}(x), f(x)$ possess a nontrivial left common divisor. (Similar result for right divisors.)

We conclude the paper with some remarks on left and right principal ideal rings with primitive images which are finite dimensional matrix rings of bounded degree over division rings.

Received by the editors May 5, 1959. 
2. Proof of Theorem 1. First we determine the structure of the primitive ideals and images of $D[x]$.

It is well known that $D[x]$ is a principal right and left ideal ring and every (two-sided) ideal $P$ in $D[x]$ has the form: $p(x) \cdot D[x]$ $=D[x] \cdot p(x)=P$, where $p(x)$ can be chosen, if not zero, to be $p(x)$ $=x^{n}+\alpha_{1} x^{n-1}+\cdots+\alpha_{n}$ with $\alpha_{i} \in$ Center $C$ of $D$ (e.g. [1, p. 38]).

The primitive ideals of $D[x]$ are the maximal ideals contained in maximal right ideals. Now, $I=f(x) D[x]$ is a maximal right ideal in $D[x]$ if and only if $f(x)$ is irreducible in $D[x]$ and it follows, therefore, by $[1$, Chapter 3 , Theorem 13] that the maximal two sided ideal $P$ contained in $I$ is either zero or a maximal ideal in $D[x]$. The latter is clearly equivalent to the fact that $P=p(x) D[x]$ is either zero or $p(x)$ is an irreducible polynomial in $C[x]$. Let $C(\xi)$ be the algebraic field extension obtained from $C$ by adjoining $\xi$-a root of the polynomial $p(x)$. Then, the isomorphism: ${ }^{1}$

$$
D[x] / p(x) D[x] \cong D \otimes C[x] / p(x) C[x] \cong D \otimes C(\xi)
$$

imply:

Lemma. Every primitive homomorphic image of $D[x]$ is either $D[x]$ or isomorphic with $D \otimes C(\xi)$ for some simple algebraic extension $C(\xi)$ of $C$.

Clearly, by ranging over all extensions $C(\xi)$ we obtain all primitive images (with the possible exception of $D[x]$ ).

We are now in position to prove Theorem 1 :

Let $(D: C) \leqq n^{2}<\infty$; then by classical results on central simple algebras (e.g. [1, Chapter 5]), $D \otimes C(\xi)$ is also a central simple algebra of dimension $\leqq n^{2}$ over its center; and, consequently, it is isomorphic with $A_{r}$ for some division algebra $A$ and for $r \leqq n$. Furthermore, in this case every nonzero right ideal contains nonzero two sided ideal $[1$, Chapter 3 , Theorem 15] which means that $D[x]$ cannot be primitive. This proves the necessity of the conditions of Theorem 1 .

To obtain the sufficiency, we consider the maximal right ideal $(x-a) D[x]=I$ for each $a \in D$. Let $P=p(x) D[x]$ be the maximal ideal contained in $I$. Since $P$ is also primitive, $D[x] / P \cong A_{r}$ with $r \leqq n$ and some division ring $A$. Our first object is to show that $\operatorname{deg} p(x) \leqq r$.

Indeed, for every $u \in D, u \neq 0$, the right ideal $\left(x-u a u^{-1}\right) D[x]=I_{u}$ contains $P$, since $u P=P \subseteq u(x-a) D[x]=I_{u}$. Furthermore, the intersection $\cap I_{u}$ for all $u \in D$ is exactly $P$, since $\cap I_{u} \supseteq P$ and $\cap I_{u}$ is clearly a two sided ideal. Now since $D[x] / P \cong A_{r}$ we can find $r$ elements $u_{1}, u_{2}, \cdots, u_{2}$ such that $D[x] \supset I_{u_{1}} \supset I_{u_{1}} \cap I_{u_{2}} \supset \cdots \supset I_{u_{1}} \cap \cdots$

${ }^{1}$ All tensor products are taken with respect to $C$. 
$\cap I_{u_{r}}=P=P$ since the length of composition chain of right ideals in $I_{r}$ is $r$. From this it follows that $p(x)$ is the left common divisors of the polynomials $x-u_{i} a u_{i}^{-1}$, and consequently deg $p(x) \leqq r$. (Actually it can be shown from the preceding chain that $\operatorname{deg} p(x)=r$, but we do not need this result.)

The completion of the proof of Theorem 1 is now as follows: from the preceding arguments it follows that for every $a \in D$ there exists a polynomial $p(x)$ of degree $\leqq n$ with coefficient in the center $C$ such that $x-a$ is a right divisor of $p(x)$. Hence, $p(a)=0$. This means that $D$ is an algebraic algebra over $C$ of degree $\leqq n$. It follows therefore by a result of Jacobson [2] that $(D: C) \leqq n^{2}$, and the proof is completed.

3. Proof of Theorem 2. It was shown in [3] that a necessary and sufficient condition for a ring $R$ that every primitive homomorphic image of $R$ is isomorphic with $A_{h}, h \leqq n$ is that $\lambda^{n}$ be a J-pivotal monomial of $R$, [3, Theorem 4]. This means that for every $r, y \in R$ there exists $s \in R$ such that:

$$
r^{n} y+s-r^{n} y s \in r^{n+1} R .
$$

In our case $R=D[x]$, this is equivalent to the fact that for every $f(x), g(x) \in D[x]$, there is $k(x) \in D[x]$ such that:

$$
f^{n}(x) g(x)+k(x)-f^{n}(x) g(x) k(x)=f^{n+1}(x) t(x)
$$

for some $t(x)$. This clearly implies that $k(x)=f^{n}(x) h(x)$, and since $D[x]$ is a ring without zero divisors, it is readily seen that the last condition is equivalent to the existence of $h(x), t(x)$ for every $g(x)$ such that:

$$
g(x)+h(x)-g(x) f^{n}(x) h(x)=f(x) t(x) .
$$

Next we show that $(J)$ is equivalent to the condition that $1-g(x) f^{n}(x)$ and $f(x)$ are relatively left prime, i.e. they do not have a nontrivial common left divisor. Indeed, if $(\mathrm{J})$ is valid then we deduce from it that: $g(x)=f(x) t(x)-\left(1-g(x) f^{n}(x)\right) h(x)$, and if $p(x)$ is a left divisor of $f(x)$ and $1-g f^{n}$ then clearly it must be also a left divisor of $g(x)$ whereas $1-g f^{n}$ and $g$ have only trivial left common divisors. Conversely, if $1-g f^{n}$ and $g$ are left prime, then since $D[x]$ is a principal right ideal ring it follows that:

$$
1=\left(1-g(x) f^{n}(x)\right) a(x)+f(x) b(x)
$$

and thus $(\mathrm{J})$ is valid with $h(x)=-a(x) g(x), t(x)=b(x) g(x)$; from the above arguments, Theorem 2 follows immediately. 
Corollary 1 follows now by a symmetrical application of right and left arguments in Theorem 2, and Corollary 2 is evident.

The arguments of the preceding section can be carried over to any principal right ideal ring $R$ without zero divisor. Namely,

THEOREM 3. Every homomorphic image of a principal right ideal ring $R$ without zero divisors is isomorphic with a complete matrix ring $A_{n}$ over a division ring if and only if for every two elements $a, b \in R$ the elements $1-b a^{n}$ and $b$ are left prime.

\section{BIBLIOGRAPHY}

1. N. Jacobson, The theory of rings, Mathematical Surveys, vol. 2, American Mathematical Society, 1943.

2. - Structure theory for algebraic algebras of bounded degree, Ann. of Math. vol. 46 (1945) pp. 695-702.

3. S. A. Amitsur, Rings with a pivotal monomial, Proc. Amer. Math. Soc. vol. 9 (1958) pp. 635-642.

HEBREW UNIVERSITY AND

UNIVERSITY OF NOTRE DAME 